\title{
RESEARCH
}

Open Access

\section{Diffusion-weighted MRI in staging of post hepatitis $C$ fibrosis: does ADC value challenge liver biopsy?}

\author{
Dena Serag and Eman Ragab* (D)
}

\begin{abstract}
Background: There is obvious interest in finding a non-invasive diagnostic tool to detect the development of hepatic fibrosis and distinguish between its various stages. Chronic inflammation of the liver secondary to viral hepatitis, autoimmune conditions, sclerosing cholangitis, drug toxicity, chronic alcohol intake, different metabolic disorders, and steatosis lead to fibrosis and maybe cirrhosis. The current study aimed to assess the usefulness of diffusion-weighted magnetic resonance imaging (DW-MRI) in diagnosis of post hepatitis $C$ fibrosis and detection of its stage.

Results: A prospective study had included 232 participants; 120 patients had chronic hepatitis C with/without HCC and 112 subjects had normal liver. There was no significant difference between the two groups regarding age or gender ( $p 0.192$ and 0.227 respectively). DW-MRI was performed using 1.5 T machine. The mean liver ADC values and normalized liver ADC (liver ADC/spleen ADC) were measured at $b$ value $800 \mathrm{~s} / \mathrm{mm}^{2}$; both were significantly lower among cases than controls. Cutoff values of liver ADC were $1.531 \times 10^{-3} \mathrm{~mm}^{2} / \mathrm{s}, 1.409 \times 10^{-3} \mathrm{~mm}^{2} / \mathrm{s}, 1.192 \times$ $10^{-3} \mathrm{~mm}^{2} / \mathrm{s}$, and $1.093 \times 10^{-3} \mathrm{~mm}^{2} / \mathrm{s}$ for METAVIR stages $\geq F 1, \geq F 2, \geq F 3$, and F4, respectively. Normalized liver ADC showed larger area under the curve (AUC) than mean liver ADC in all differentiation categories except for differentiating between F0 and all other fibrosis stages.

Conclusion: In line with the literature, DW-MR imaging using $b$ value of $800 \mathrm{~s} / \mathrm{mm}^{2}$ has proved to be a valuable diagnostic technique for detection and staging of post hepatitis $C$ fibrosis/cirrhosis being noninvasive procedure with acceptable accuracy. DWI using liver/spleen ADC values raised the diagnostic performance with AUC more than $90 \%$ in all fibrosis stages on METAVIR score.
\end{abstract}

Keywords: Liver fibrosis, Cirrhosis, Diffusion magnetic resonance imaging, Hepatitis C

*Correspondence: emanragab2000@yahoo.com

Department of Radiodiagnosis, Faculty of Medicine, Menoufia University, Menoufia 32511, Egypt

(c) The Author(s). 2020 Open Access This article is licensed under a Creative Commons Attribution 4.0 International License, which permits use, sharing, adaptation, distribution and reproduction in any medium or format, as long as you give appropriate credit to the original author(s) and the source, provide a link to the Creative Commons licence, and indicate if changes were made. The images or other third party material in this article are included in the article's Creative Commons licence, unless indicated otherwise in a credit line to the material. If material is not included in the article's Creative Commons licence and your intended use is not permitted by statutory regulation or exceeds the permitted use, you will need to obtain permission directly from the copyright holder. To view a copy of this licence, visit http://creativecommons.org/licenses/by/4.0/. 


\section{Background}

Chronic liver disease represents a common health problem with many etiologies, primarily viral infection by hepatitis $\mathrm{B}$ and $\mathrm{C}$ viruses. Liver fibrosis acts as a repair process responding to the chronic hepatic injury whatever the cause. Unfortunately, in most cases, it progresses to liver cirrhosis, which poses a serious threat to human health by its subsequent complications [1]. It is so vital to diagnose fibrosis before developing early or advanced cirrhosis.

Liver biopsy is the gold standard for staging fibrosis and assessment of inflammatory necrotic changes [2, 3], yet it is an invasive tool and has underlying risk of bleeding. In addition, biopsy is limited by inter-observer variance and sampling fallacy [4]. Development of noninvasive and accurate alternatives was necessary, e.g., transient elastography (fibroscan) and magnetic resonance (MR) elastography to diagnose liver fibrosis and its stage [5]. Latterly, use of diffusion-weighted MR imaging in assessment and staging of liver fibrosis has been encountered [6].

Diffusion-weighted imaging (DWI) is a developed technique of magnetic resonance imaging (MRI) used specifically in assessment of the microscopic construct of the tissues. It depends on the water molecules' motion within the tissues [7]. Many studies had designated the efficacy of quantitative apparent diffusion coefficient (ADC) measurement by this imaging technique in liver fibrosis [2]. ADC is the most frequently used DWI parameter that allows valuable idea about inflammation, tissue perfusion, and local cell breakdown. Previous researches have declared that water diffusion may be reduced by extracellular collagen deposition and proteoglycans in cases of liver fibrosis, therefore, such cases reported lower ADC values [6].

Our present study aimed to evaluate DWIs as a noninvasive technique to diagnose liver fibrosis and its stage compared to the liver biopsy and histopathological correlation.

\section{Methods}

\section{Patients and control group}

This prospective study was conducted in the period between October 2017 and March 2020 with participation of 120 patients, 84 males (70\%) and 36 females (30\%), age range $32-77$ years with mean \pm SD $57.48 \pm 9.95$. The included patients were proved to have chronic hepatitis $C$ by polymerase chain reaction (PCR) elicited during a national screening program. Excluded patients were those with concomitant positive hepatitis B surface antigen, patients with positive antinuclear antibody, patients with illness since childhood and probability of metabolic disorders, and patients on long-term chemotherapy. Fifty cases with hepatocellular carcinoma
(HCC) representing $41.67 \%$ and 70 cases without $\mathrm{HCC}$ representing $58.33 \%$.

A control group of 112 was assumed to have healthy liver with normal laboratory and imaging findings; excluding those with fatty liver and whom had any hepatic focal lesions either cystic or solid in nature (70 males $(62.5 \%)$ and 42 females (38.5\%), age range $45-67$ years with mean \pm SD $58.93 \pm 6.49$ ).

Liver biopsies were taken ultrasound guided in the day after the MRI procedure using 18-gage core needle. An interventional radiologist of more than 5 years' experience in organ core biopsies did this work for all patients in the ultrasonography unit. The Institutional Research Ethics committee had approved the study with a written informed consent from each case.

\section{Examination technique and image analysis}

Using a 1.5-T closed MR system (Siemens Magnetom Aera); complete dynamic protocol was performed using the body coil. The DWI images were obtained with these parameters: TR 5500/TE 62.0/slice thickness $6.5 \mathrm{~mm} /$ inter-slice gap $3.25 \mathrm{~mm} / \mathrm{FOV} 306 \times 380 \mathrm{~mm} /$ Matrix $216 \times 268 /$ number of excitations $2 /$ b1-values of $400 / 90$ / b2 $800 / 90 \mathrm{~s} / \mathrm{mm}^{2}$.

The images were sent to the workstation (NUMARIS/ 4 syngo MR E11 4VE11C) where ADC values were calculated by locating 6 rounded regions of interest (ROIs) approximately $6 \mathrm{~cm}^{2}$ as follows: two ROIs in the right lobe of the liver, another two ROIs in the left lobe, and the last two ROIs were in the spleen excluding the organ vessels and images with artifacts as shown in (Figs. 1, 2, 3, and 4). The mean ADC of both hepatic lobes represented liver $\mathrm{ADC}$, and ratio of this $\mathrm{ADC}$ value to the mean spleen $\mathrm{ADC}$ value was the normalized liver ADC using the spleen as a reference organ.

The measurement of ADC values was calculated by a radiologist of more than 12 years' experience with nearly standardized location of each ROI from the start.

The liver biopsies were reported using the METAVIR score as follows: F0 = no fibrosis, F1 = portal fibrosis, F2 = periportal fibrosis, F3 = septal fibrosis, and F4 = cirrhosis $[8,9]$. Each histopathological interpretation was performed by two pathologists of more than 10 years' experience with a higher consultation by a professor of pathology of more than 20 years' experience using a multi-head microscope.

\section{Statistical analysis}

Data were analyzed using SPSS statistical package version 23 (SPSS Inc. Released 2015. IBM SPSS statistics for windows, version 23.0, Armonk, NY: IBM Corp). 


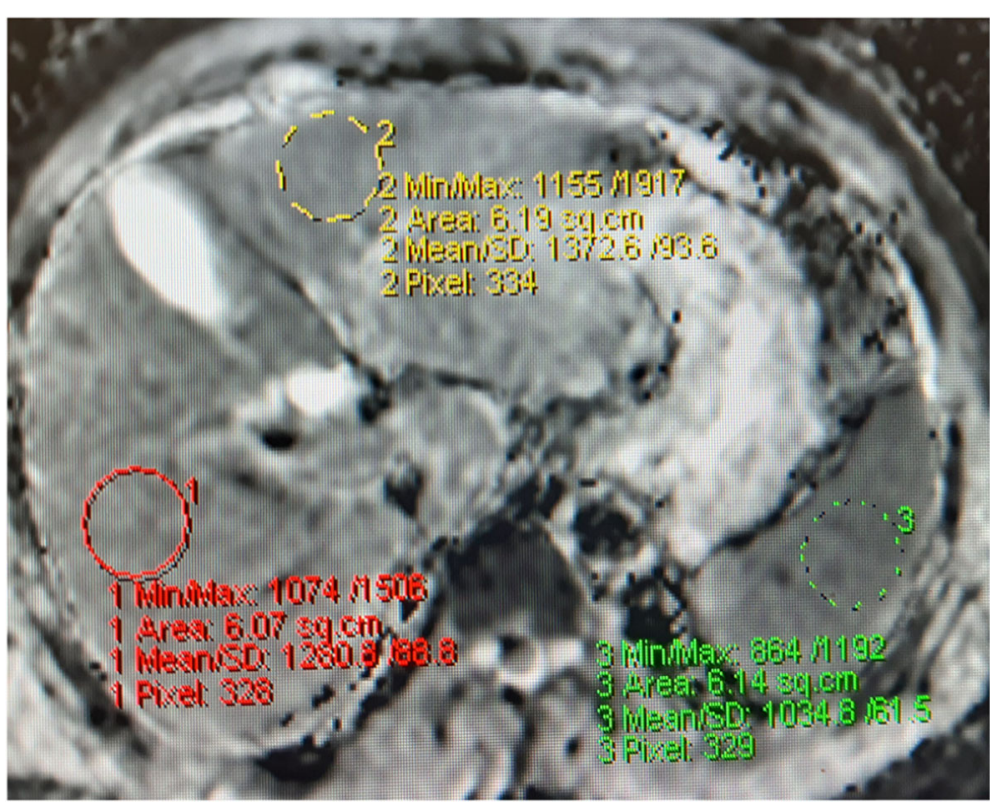

Fig. 1 DW-MR $(b=800)$ image and ADC map of the liver and the spleen for a case of F2 stage [mean liver ADC value $=1376.3 \mathrm{~mm}^{2} / \mathrm{s}$, mean spleen $A D C$ value $=1009.6 \mathrm{~mm}^{2} / \mathrm{s}$, and liver $/$ spleen $A D C=1.36$ ]

- Tests for significance of the differences between two groups were Student's $t$ test and Mann Whitney's test.

- For more than two groups, ANOVA test with Tukey test and Kruskal Wallis test with Tamhane's test were used.

- Chi-square test ( $\mathrm{x} 2)$ was used to study association between qualitative variables. Whenever any of the expected cells were less than five, Fisher's exact test was used.

- Receiver operator characteristic (ROC) with respective points of maximal accuracy for sensitivity and specificity were generated to determine biomarker performance.

- Two-sided $P$ value of $<0.05$ was considered statistically significant.

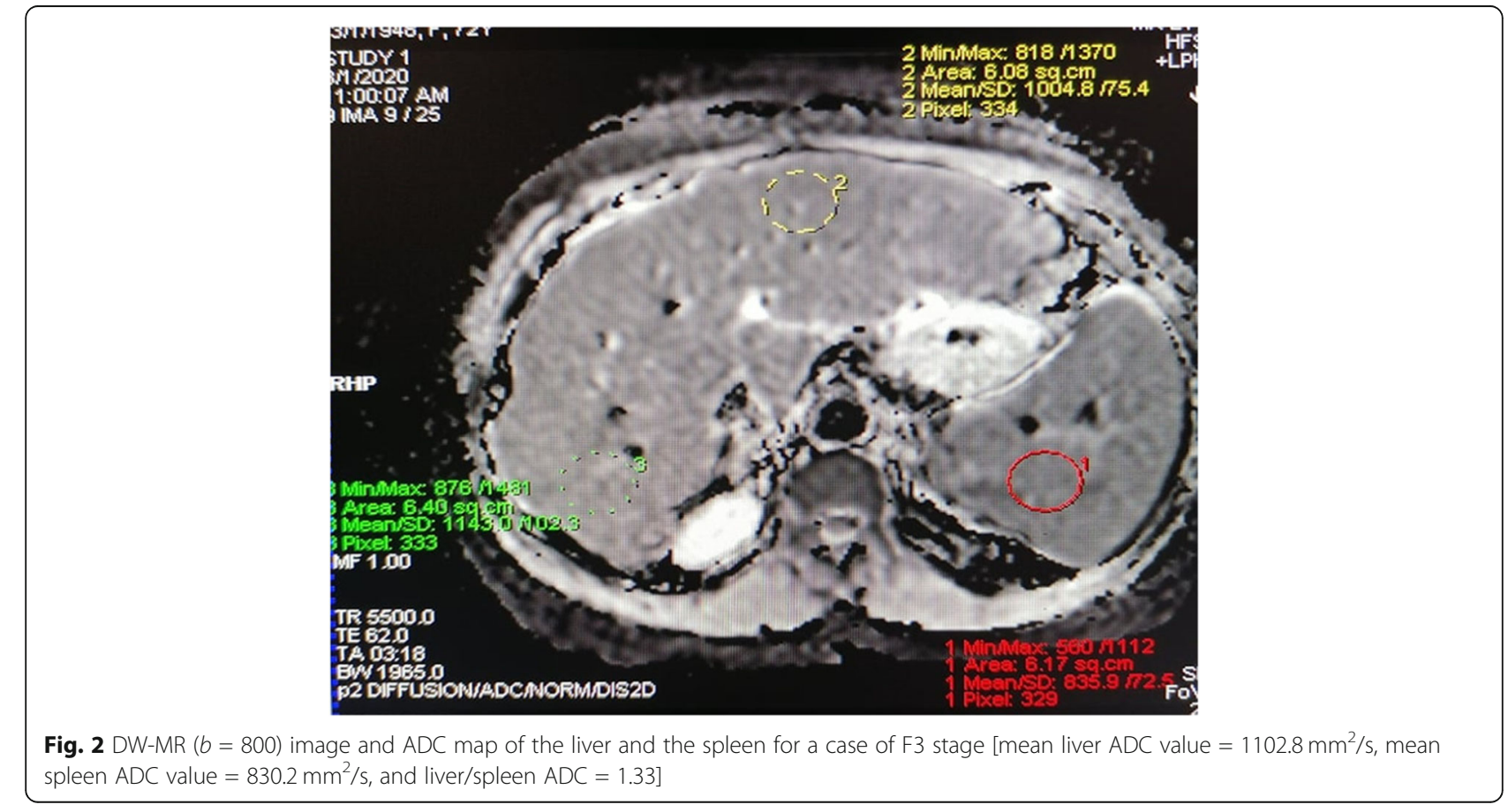




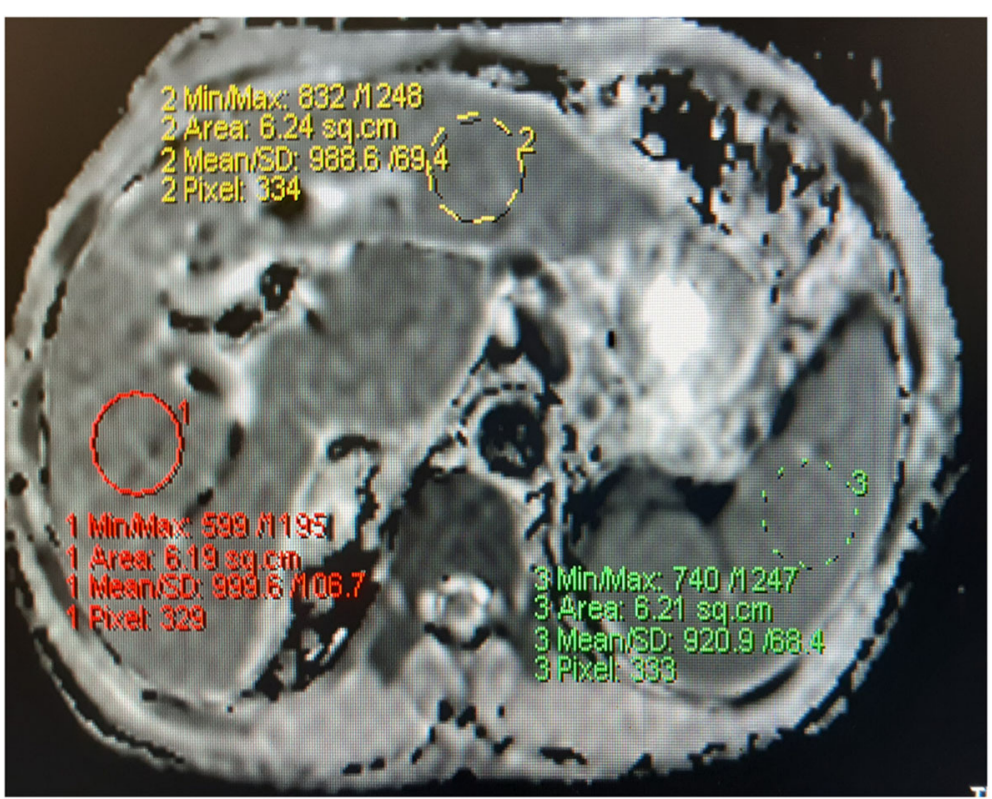

Fig. 3 DW-MR $(b=800)$ image and ADC map of the liver and the spleen for a case of F4 stage [mean liver ADC value $=999.8 \mathrm{~mm}^{2} / \mathrm{s}, \mathrm{mean}$ spleen $A D C$ value $=915 \mathrm{~mm}^{2} / \mathrm{s}$, and liver $/$ spleen $A D C=1.09$ ]

\section{Results}

A total of 232 participants were included in this study; 120 patients had chronic hepatitis $\mathrm{C}$ with/without HCC, and 112 subjects had normal liver. There was no significant difference between the two groups regarding age or gender ( $p 0.192$ and 0.227 respectively). The mean liver
$\mathrm{ADC}$ values and normalized liver $\mathrm{ADC}$ (liver $\mathrm{ADC} /$ spleen ADC) were both significantly lower among cases than controls, yet no significant difference was noted regarding the spleen ADC values, $p 0.618$ (Table 1).

Regarding the histopathological results, according to the METAVIR scoring system, 21 cases were of stage F1,

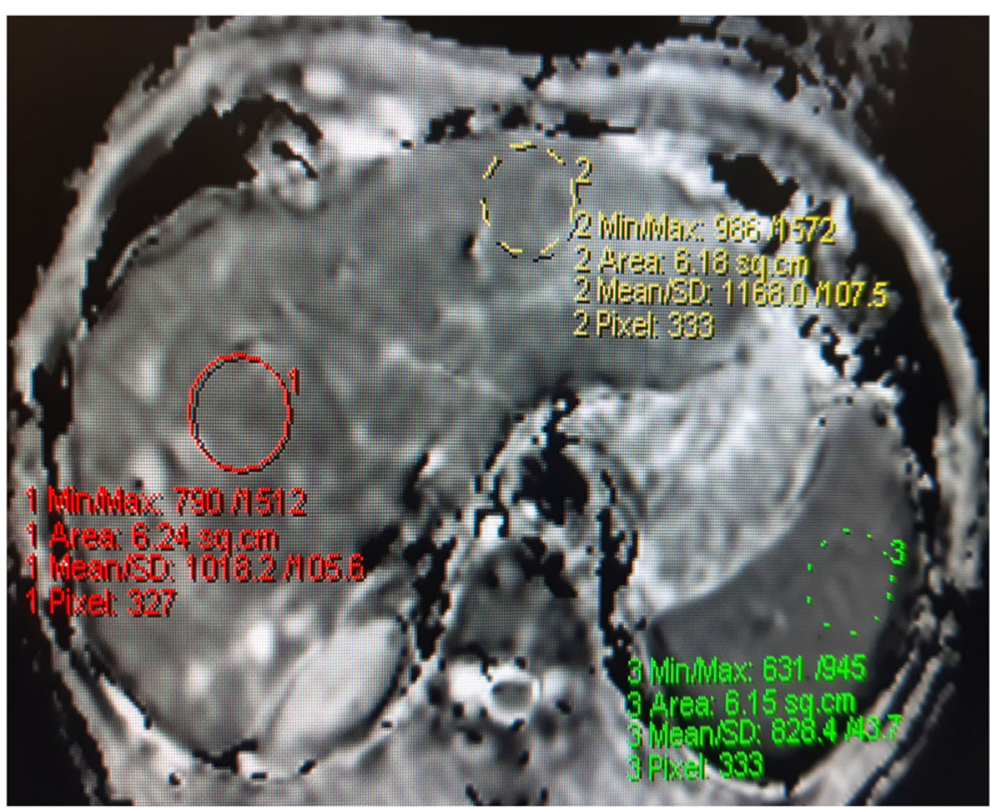

Fig. 4 DW-MR $(b=800)$ image and ADC map of the liver and the spleen for a case of F4 stage with HCC [mean liver ADC value $=1093.5 \mathrm{~mm}{ }^{2} / \mathrm{s}$, mean spleen $A D C$ value $=868.4 \mathrm{~mm}^{2} / \mathrm{s}$, and liver/spleen $\left.A D C=1.26\right]$ 
Table 1 Age and different ADC measurements between cases and control group

\begin{tabular}{llll}
\hline Variable & Cases $(n=120)$ mean \pm SD & Controls $(n=112)$ mean \pm SD & $P$ value \\
\hline Age & $57.48 \pm 9.95$ & $58.93 \pm 6.49$ & 0.192 \\
Liver ADC & $1142.96 \pm 158.76$ & $1659.43 \pm 101.71$ & $<0.001$ \\
Spleen ADC & $806.52 \pm 100.36$ & $813.18 \pm 101.40$ & 0.618 \\
Liver/spleen ADC (normalized liver ADC) & $1.43 \pm 0.25$ & $2.07 \pm 0.27$ & $<0.001$ \\
\hline
\end{tabular}

40 cases were of stage F2, 36 cases were of stage F3, and finally 23 cases were of stage F4. There was no significant difference regarding age among patients with different stages of fibrosis. There were significant differences regarding the measurements of the liver ADC and normalized liver ADC values among patients with different stages of fibrosis. Patients with F1 had the highest measures followed by F2 followed by F3 and finally F4 had the lowest measurements, with significant difference among each other as elicited in Table 2.

The mean liver ADC and normalized liver ADC showed significantly larger measurements among patients with stage F1 than all other fibrosis stages. Patients with stages F2 and F3 had significantly larger measurements than those of stage F4, with no significant difference was found between grades F2 and F3.

Among the case groups $(n=120)$, there was no significant difference between patients with and patients without $\mathrm{HCC}$ regarding the age, liver ADC, spleen ADC, and normalized liver ADC as shown in Table 3.

There was no significant difference between patients with mild (F1 and F2) fibrosis and severe (F3 and F4) fibrosis regarding age ( $p$ 0.0151). Spleen ADC was significantly smaller $(p<0.001)$ while liver ADC and normalized liver ADC were significantly larger in patients with mild fibrosis than severe fibrosis $(p 0.001$ and $<0.001$ respectively) as shown in Table 4 .

The ROC curve analysis for liver ADC and normalized liver ADC showed good performance of both measures. Mean liver ADC best performed in differentiating between F0 (control group) and all other fibrosis groups. Normalized liver ADC best performed in differentiating between F4 and all other fibrosis grades. Normalized liver ADC showed larger area under the curve (AUC) than mean liver ADC in all differentiation categories except for differentiating between F0 and all other fibrosis stages as demonstrated in Table 5, Figs. 5 and 6.

The diagnostic accuracy of liver ADC and liver/ spleen ADC (normalized liver ADC) to assess stage of liver fibrosis compared to the histopathological correlation revealed that both parameters were significant predictors in staging. For example, using liver ADC, $\geq$ F1 stage with $b$ value $800 \mathrm{~s} / \mathrm{mm}^{2}$, AUC, sensitivity, specificity, and accuracy were 0.987, 96.6\%, 87.4\%, and $92 \%$ respectively, the cut off $\mathrm{ADC}$ value was $1.531 \times 10^{-3} \mathrm{~mm}^{2} / \mathrm{s}$. Using liver/spleen ADC for the same category, AUC, sensitivity, specificity, and accuracy were $0.957,94.9 \%, 80.2 \%$, and $88 \%$ respectively; the cutoff value was 1.844 .

For F4 stage with liver ADC, AUC, sensitivity, specificity, and accuracy were $0.940,87 \%, 85.4 \%$, and $86 \%$ respectively; the cutoff $\mathrm{ADC}$ value was $1.093 \times$ $10^{-3} \mathrm{~mm}^{2} / \mathrm{s}$. Using liver/spleen ADC for the same category, AUC, sensitivity, specificity, and accuracy were $0.986,95.7 \%, 89.3 \%$, and $90 \%$ respectively. 1.355 was the cutoff value.

\section{Discussion}

Liver fibrosis is considered a major morbidity that can lead to serious complications like portal hypertension

Table 2 Age and different ADC measurement of different fibrosis stages among cases

\begin{tabular}{|c|c|c|c|c|c|c|}
\hline Variable & $\mathrm{F} 1(n=21)$ mean $\pm \mathrm{SD}$ & $\mathrm{F} 2(n=40)$ mean $\pm \mathrm{SD}$ & $\mathrm{F} 3(n=36)$ mean $\pm \mathrm{SD}$ & $\mathrm{F} 4(n=23)$ mean $\pm \mathrm{SD}$ & $P$ value & Post hoc \\
\hline Age (year) & $54.52 \pm 12.78$ & $57.07 \pm 8.44$ & $56.63 \pm 10.19$ & $62.21 \pm 7.93$ & 0.059 & --- \\
\hline Liver ADC & $1280.33 \pm 140.95$ & $1139.55 \pm 133.05$ & $1158.23 \pm 159.35$ & $999.56 \pm 81.09$ & $<0.001$ & $\begin{array}{l}P 1<0.001 \\
P 20.001 \\
P 3<0.001 \\
P 40.549 \\
P 5<0.001 \\
P 6<0.001\end{array}$ \\
\hline $\begin{array}{l}\text { Liver/spleen ADC } \\
\text { (normalized liver ADC) }\end{array}$ & $1.74 \pm 0.26$ & $1.46 \pm 0.13$ & $1.41 \pm 0.17$ & $1.12 \pm 0.12$ & $<0.001$ & $\begin{array}{l}P 10.001 \\
P 2<0.001 \\
P 3<0.001 \\
P 40.684 \\
P 5<0.001 \\
P 6<0.001\end{array}$ \\
\hline
\end{tabular}


Table 3 Age and different ADC measurements between patients with and without HCC among the cases group

\begin{tabular}{llll}
\hline Variable & With HCC $(n=50)$ mean \pm SD & Without HCC $(n=70)$ mean \pm SD & $P$ value \\
\hline Age & $56.34 \pm 11.16$ & $59.08 \pm 7.79$ & 0.138 \\
Spleen & $803.14 \pm 107.15$ & $811.45 \pm 90.40$ & 0.660 \\
Liver ADC & $1150.43 \pm 181.01$ & $1132.50 \pm 121.98$ & 0.544 \\
Liver/spleen ADC & $1.45 \pm 0.30$ & $1.40 \pm 0.16$ & 0.547 \\
\hline
\end{tabular}

and hepatic cellular failure [10]; thus, it is more wisely to be managed in early stages. It is crucial to determine the stage of hepatic fibrosis as antiviral therapy will be beneficial to cases with $\geq$ F2 [11].

Previous researches had reported a relationship between the liver fibrosis and ADC values. The current study used $b$ values 400 and $800 \mathrm{~s} / \mathrm{mm}^{2}$. Taouli et al. [2] stated a significant correlation on using $b$ values of $\geq$ $500 \mathrm{~s} / \mathrm{mm}^{2}$ and Jiang et al. [12], a meta-analysis concluded that DWI of the liver is a reliable noninvasive diagnostic tool for liver fibrosis staging using $b_{\max } \geq 800$ $\mathrm{s} / \mathrm{mm}^{2}$.

The literature stated that DW-MRI had demonstrated lower ADC values in liver fibrosis than normal liver [1, $2,12-20]$. In concordance with the previous researches, the liver ADC values of the cases were significantly lower than the ADC values of the control group. Regarding the diagnostic performance in distinguishing different stages of fibrosis, Do et al. [21] had used $b$ values of 0,50 , and $500 \mathrm{~s} / \mathrm{mm}^{2}$ and reported cutoff values of $1.68 \times 10^{-3} \mathrm{~mm}^{2} / \mathrm{s}, 1.53 \times 10^{-3} \mathrm{~mm}^{2} / \mathrm{s}$, and $1.68 \times 10^{-3}$ $\mathrm{mm}^{2} / \mathrm{s}$ for METAVIR stages $\geq F 2, \geq \mathrm{F} 3$, and F4, respectively. Their study group was smaller (34 patients) and inhomogeneous including many etiologies rather than viral hepatitis $\mathrm{C}$.

Lower values for the same stages have been elicited in our study $1.409 \times 10^{-3} \mathrm{~mm}^{2} / \mathrm{s}, 1.192 \times 10^{-3} \mathrm{~mm}^{2} / \mathrm{s}$, and $1.093 \times 10^{-3} \mathrm{~mm}^{2} / \mathrm{s}$ for METAVIR stages $\geq \mathrm{F} 2, \geq$ $\mathrm{F} 3$, and F4, respectively using $b$ values of 400 and $800 \mathrm{~s} / \mathrm{mm}^{2}$. Bonekamp et al. [18] have demonstrated cutoff values of $1.33 \times 10^{-3} \mathrm{~mm}^{2} / \mathrm{s}, 1.31 \times 10^{-3} \mathrm{~mm}^{2} /$ $\mathrm{s}$, and $1.30 \times 10^{-3} \mathrm{~mm}^{2} / \mathrm{s}$ to detect METAVIR stages $\geq \mathrm{F} 2, \geq \mathrm{F} 3$, and $\mathrm{F} 4$, respectively using $b$ values 0 and $750 \mathrm{~s} / \mathrm{mm}^{2}$. The number of cases belonging to F2 and F3 stages was notably small (2 cases for F2 and 6 cases for F3). Variable $b$ values and different sample sizes as well as the patient's characterization regarding different causative entities might explain the difference.

Shayesteh et al. [20] with $b$ value of $1000 \mathrm{~s} / \mathrm{mm}^{2}$ and $1.5 \mathrm{~T}$ scanner have reported somehow similar cutoff values $1.223 \times 10^{-3} \mathrm{~mm}^{2} / \mathrm{s}, 1.186 \times 10^{-3} \mathrm{~mm}^{2} / \mathrm{s}$, and $1.140 \times 10^{-3} \mathrm{~mm}^{2} / \mathrm{s}$ to detect METAVIR stages $\geq \mathrm{F} 2, \geq$ F3, and F4, respectively. Also, the AUC 0.908, 0.889, and 0.933 for $\geq$ F2, $\geq$ F3, and F4 respectively.

Similarly, Fujimoto et al. [16] with $b$ values of 0 and $1000 \mathrm{~s} / \mathrm{mm}^{2}$ have declared close cutoff values of $1.35 \times$ $10^{-3} \mathrm{~mm}^{2} / \mathrm{s}$ (METAVIR $\geq$ F1), $1.32 \times 10^{-3} \mathrm{~mm}^{2} / \mathrm{s}$ (METAVIR $\geq$ F2), $1.27 \times 10^{-3} \mathrm{~mm}^{2} / \mathrm{s}($ METAVIR $\geq$ F3), and $1.23 \times 10^{-3} \mathrm{~mm}^{2} / \mathrm{s}$ (METAVIR F4).

It is believed that significant periportal fibrosis (F2 stage) is considered a predictor of cirrhosis; thus, the aim of diagnosis and treatment during this stage is to manage the underlying etiology and abort its effect. Besides, high accuracy in the diagnosis of severe fibrosis (F3 and F4) is important, as these patients should be followed up and screened for development of portal hypertension and HCC [22, 23].

Regarding the values of ADC liver for cirrhosis (stage F4 by METAVIR score), our study reported $999.56 \pm$ $81.09 \mathrm{~mm}^{2} / \mathrm{s}$ (mean $\pm \mathrm{SD}$ ) for 23 cases. Close result was obtained by Verloh et al. [24] $1015 \pm 60.2 \mathrm{~mm}^{2} / \mathrm{s}$ for the same pathological category. But they used another scoring system instead of METAVIR (Ishak score) and a 3 T MRI system.

Again, similar values concerning F4 stage were recorded by $\mathrm{Hu}$ et al. [1], whom used variable $b$ values and $1.5 \mathrm{~T}$ scanner. With $b$ value $700 \mathrm{~s} / \mathrm{mm}^{2}$ and another scoring system similar to METAVIR, they reported $1150 \pm 22 \mathrm{~mm}^{2} / \mathrm{s}$.

Our study revealed relatively larger area under the curve (AUC) of normalized ADC liver in diagnosis of

Table 4 Age and different ADC measurements between cases with mild fibrosis and severe fibrosis

\begin{tabular}{llll}
\hline Variable & Mild fibrosis $(F 1$ and F2) $(n=61)$ mean \pm SD & Severe fibrosis $(F 3$ and F4) $(n=59)$ mean \pm SD & $P$ value \\
\hline Age & $56.19 \pm 10.11$ & $58.81 \pm 9.69$ & 0.151 \\
Spleen ADC & $764.27 \pm 95.09$ & $848.77 \pm 87.41$ & $<0.001$ \\
Liver ADC & $1188.01 \pm 150.59$ & $1096.38 \pm 154.62$ & 0.001 \\
Liver/spleen ADC & $1.56 \pm 0.23$ & $1.30 \pm 0.21$ & $<0.001$ \\
\hline
\end{tabular}


Table 5 Comparison between mean liver ADC and normalized liver ADC regarding the diagnostic performance and cutoff values

\begin{tabular}{|c|c|c|c|c|c|c|c|}
\hline Class & Cutoff & AUC & Sensitivity & Specificity & PPV & NPV & Accuracy \\
\hline \multicolumn{8}{|c|}{ Mean liver ADC } \\
\hline $\mathrm{F} 0 \mathrm{vs} \geq \mathrm{F} 1$ & 1531.0 & 0.987 & 96.6 & 87.4 & 89 & 96 & 92 \\
\hline$\leq F 1$ vs $\geq F 2$ & 1409.75 & 0.968 & 92.8 & 85.6 & 83 & 94 & 89 \\
\hline$\leq F 2$ vs $\geq F 3$ & 1192.25 & 0.879 & 88.1 & 80.6 & 61 & 95 & 83 \\
\hline$\leq \mathrm{F} 3$ vs F4 & 1093.0 & 0.940 & 87.0 & 85.4 & 40 & 98 & 86 \\
\hline \multicolumn{8}{|c|}{ Normalized liver ADC } \\
\hline $\mathrm{F} 0 \mathrm{vs} \geq \mathrm{F} 1$ & 1.844 & 0.957 & 94.9 & 80.2 & 84 & 94 & 88 \\
\hline$\leq F 1$ vs $\geq F 2$ & 1.705 & 0.973 & 96.9 & 87.1 & 85 & 97 & 87 \\
\hline$\leq F 2$ vs $\geq F 3$ & 1.499 & 0.933 & 88.1 & 85.9 & 68 & 95 & 86 \\
\hline$\leq \mathrm{F} 3$ vs F4 & 1.355 & 0.986 & 95.7 & 89.3 & 50 & 99 & 90 \\
\hline
\end{tabular}

significant fibrosis ( $\geq$ F2) and cirrhosis (F4) compared to ADC liver; 0.973 and 0.986 compared to 0.968 and 0.940 for $\geq$ F2 and F4 stages respectively. Thus, it can be considered an excellent diagnostic tool with AUC $>90 \%[25]$.

Shin et al. [26] compared the diagnostic performance of ADC liver and normalized ADC liver and reported evident difference between them in diagnosing all fibrosis stages, for $\geq$ F2 0.631AUC, 83.4\% sensitivity, $58.5 \%$ specificity, and optimal cutoff value was $1.332 \times 10^{-3} \mathrm{~mm}^{2} / \mathrm{s}$ using liver ADC. Normalized ADC liver for the same category revealed 0.877 AUC, $84.3 \%$ sensitivity, $86.9 \%$ specificity, and optimal cutoff value was 1.411 .

For F4 stage, AUC 0.577, 43.4\% sensitivity, 83.1\% specificity, and optimal cutoff value was $1.189 \times 10^{-3}$ $\mathrm{mm}^{2} / \mathrm{s}$ using liver ADC. Normalized ADC liver for the same category revealed AUC 0.789, 90.2\% sensitivity, $62.3 \%$ specificity, and optimal cutoff value was 1.365 . This might be contributed to variable $b$ values used, and their study group was inhomogeneous including only 3 cases of post hepatitis C fibrosis.

Again, Do et al. [21] have concluded that normalization of liver ADC using the spleen as a reference organ increased the diagnostic ability for hepatic fibrosis. There was larger AUC for normalized ADC liver in all fibrosis stages $0.864,0.805$, and 0.935 for $\geq F 2$, $\geq F 3$, and F4, respectively, compared to $0.655,0.689$, and 0.720 for the same fibrosis categories using the liver ADC. Their sample was heterogeneous in nature as other causes of hepatic fibrosis rather than chronic hepatitis $\mathrm{C}$ were included. Also different $b$ values, as they used 0,50 , and $500 \mathrm{~s} / \mathrm{mm}^{2}$ $b$ values on $1.5 \mathrm{~T}$ machine.
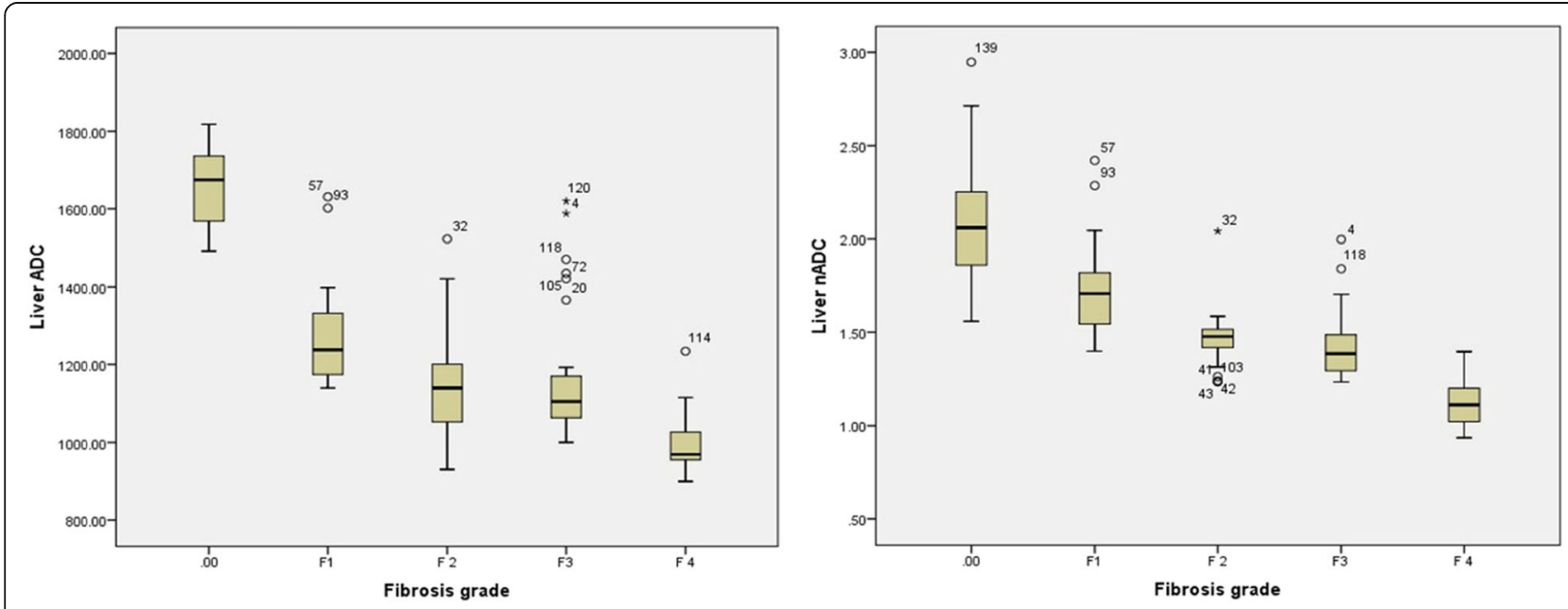

Fig. 5 Box plots showing values of ADC liver and normalized liver ADC for each stage of hepatic fibrosis. There were 112 control cases representing F0 with 21, 40, 36, and 23 patients with fibrosis stages F1, F2, F3, and F4, respectively 

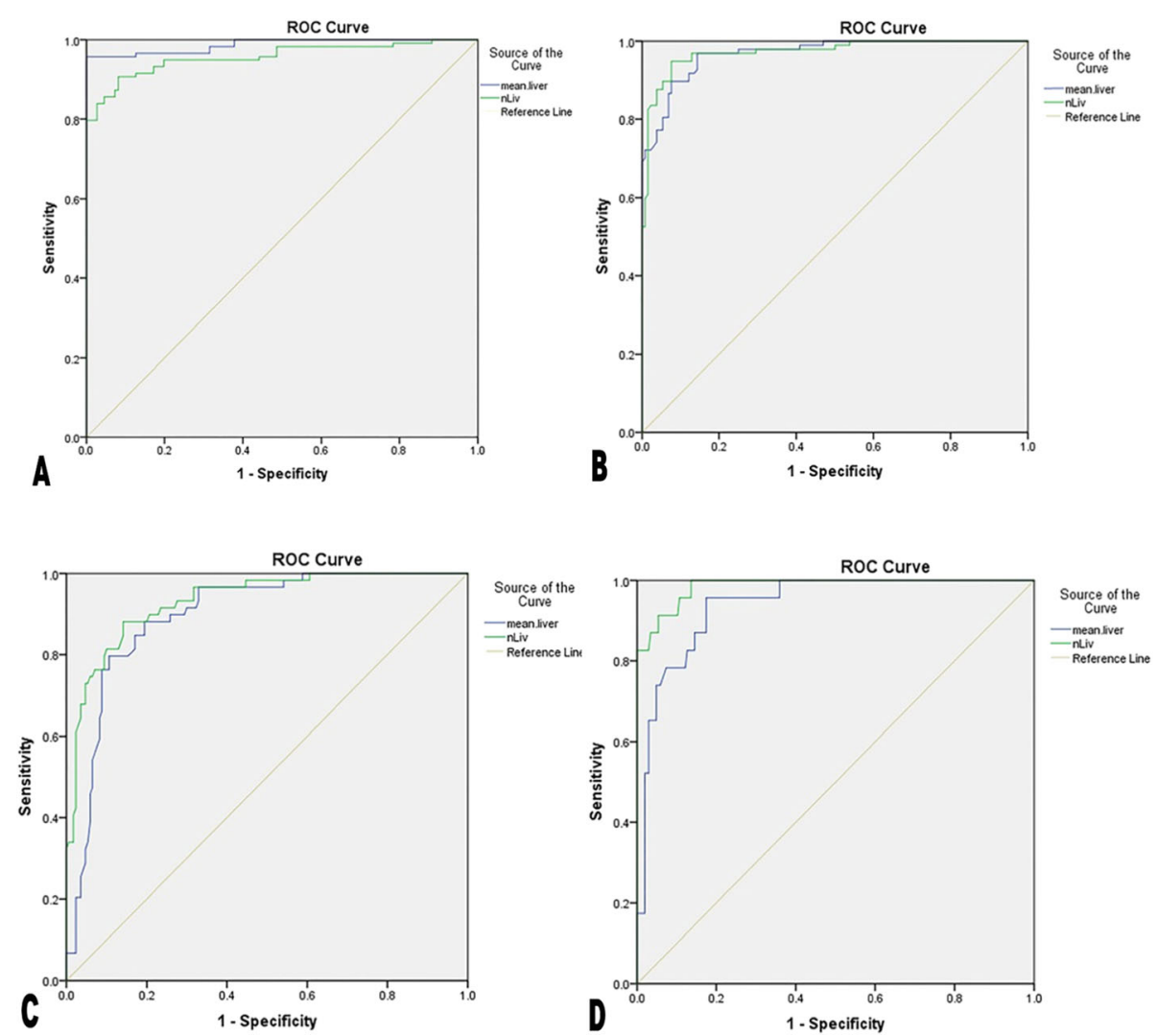

Fig. 6 Receiver operating characteristic (ROC) curve for liver ADC and normalized liver ADC in diagnosis of fibrosis stage $\geq$ F1 (a), $\geq$ F2 (b), $\geq$ F3 (c), and F4 (d)

\section{Limitations of the study}

Lack of confirmed F0 stage by histopathological correlation as the control group was included based on clinical, laboratory, and imaging findings. Lack of a standardized parameters for image acquisition.

\section{Conclusion}

In line with the literature, DW-MR imaging using $b$ value of $800 \mathrm{~s} / \mathrm{mm}^{2}$ has proved to be a valuable diagnostic technique for detection and staging of post hepatitis $\mathrm{C}$ fibrosis/cirrhosis being noninvasive procedure with acceptable accuracy. DWI using liver/spleen ADC values raised the diagnostic performance with AUC more than $90 \%$ in all fibrosis stages on METAVIR score, thus could be considered an excellent diagnostic tool. Further studies are recommended to establish an optimized imaging protocol concerning this issue.

\section{Abbreviations}

DW-MRI: Diffusion-weighted magnetic resonance imaging; ADC: Apparent diffusion coefficient; HCC: Hepatocellular carcinoma; PCR: Polymerase chain reaction; ROls: Regions of interest; ROC: Receiver operating characteristics; AUC: Area under the curve

\section{Acknowledgements}

The authors like to thank their colleagues in the pathology department and GIT \& hepatology unit. Their efforts are highly appreciated.

\section{Authors' contributions}

ER and DS contributed equally to the study design, data collection, analysis, and interpretation of results. All authors read and approved the final manuscript.

\section{Funding}

Nil.

\section{Availability of data and materials}

Data will be available upon request via contacting the corresponding author.

Ethics approval and consent to participate

A written consent to participate is available.

Ethics approval: by Menoufia University, Faculty of Medicine, Research Ethics Committee

The reference number is 3/2020RAD3.

\section{Consent for publication}

A written consent for publication is available.

\section{Competing interests}

The authors declare that they have no competing interests. 
Received: 29 May 2020 Accepted: 9 August 2020

Published online: 07 September 2020

\section{References}

1. Hu XR, Cui XN, Hu QT et al (2014) Value of MR diffusion imaging in hepatic fibrosis and its correlations with serum indices. Am J Roentgenol 20:79647970. https://doi.org/10.3748/wjg.v20.i24.7964

2. Taouli B, Tolia AJ, Losada M et al (2007) Diffusion-weighted MRI for quantification of liver fibrosis: preliminary experience. Am J Roentgenol 189: 799-806. https://doi.org/10.2214/AJR.07.2086

3. Sandrasegaran K, Akisik FM, Lin C et al (2014) Value of diffusion-weighted MRI for assessing liver fibrosis and cirrhosis. Am J Roentgenol 195:671-676. https://doi.org/10.1038/jid.2014.371

4. Cholongitas E, Senzolo M, Standish R et al (2006) A systematic review of the quality of liver biopsy specimens. Am J Clin Pathol 125:710-721. https://doi. org/10.1309/w3xc-nt4h-kfbn-2g0b

5. Godfrey EM, Mannelli L, Griffin N et al (2013) Magnetic resonance elastography in the diagnosis of hepatic fibrosis. Semin Ultrasound CT MRI 34:81-88. https://doi.org/10.1053/j.sult.2012.11.007

6. Kocakoc E, Bakan AA, Poyrazoglu OK et al (2015) Assessment of liver fibrosis with diffusion-weighted magnetic resonance imaging using different bvalues in chronic viral hepatitis. Med Princ Pract 24:522-526. https://doi.org/ 10.1159/000434682

7. Laun FB, Fritzsche KH, Kuder TA et al (2011) Introduction to the basic principles and techniques of diffusion-weighted imaging. Radiologe 51:170179. https://doi.org/10.1007/s00117-010-2057-y

8. Huang Y, Bastiaan De Boer W, Adams LA et al (2014) Image analysis of liver biopsy samples measures fibrosis and predicts clinical outcome. J Hepatol 61:22-27. https://doi.org/10.1016/j.jhep.2014.02.031

9. Poynard T, Vergniol J, Ngo $Y$ et al (2014) Staging chronic hepatitis C in seven categories using fibrosis biomarker (FibroTestTM) and transient elastography (FibroScan ${ }^{\circledast}$ ). J Hepatol 60:706-714. https://doi.org/10.1016/j. jhep.2013.11.016

10. Koinuma M, Ohashi I, Hanafusa K et al (2005) Apparent diffusion coefficient measurements with diffusion-weighted magnetic resonance imaging for evaluation of hepatic fibrosis. J Magn Reson Imaging 22:80-85. https://doi. org/10.1002/jmri.20344

11. Kim Al, Saab S (2005) Treatment of hepatitis C. Am J Med 118:808-815

12. Jiang $H$, Chen J, Gao R et al (2017) Liver fibrosis staging with diffusionweighted imaging: a systematic review and meta-analysis. Abdom Radiol 42:490-501. https://doi.org/10.1007/s00261-016-0913-6

13. Namimoto T, Yamashita Y, Sumi S et al (1997) Focal liver masses: characterization with diffusion-weighted echo-planar MR imaging. Radiology 204:739-744. https://doi.org/10.1148/radiology.204.3.9280252

14. Sandrasegaran K, Akisik FM, Lin C et al (2009) Value of diffusion-weighted MRI for assessing liver fibrosis and cirrhosis. Am J Roentgenol 193:15561560. https://doi.org/10.2214/AJR.09.2436

15. Sandrasegaran K, Akisik FM, Lin C et al (2010) Diagnosis of liver fibrosis and cirrhosis with diffusion-weighted imaging: value of normalized apparent diffusion coefficient using the spleen as reference organ. Am J Roentgenol 195:7964-7970. https://doi.org/10.1038/jid.2014.371

16. Fujimoto K, Tonan T, Azuma S et al (2011) Evaluation of the mean and entropy of apparent diffusion coefficient values in chronic hepatitis $C$ : correlation with pathologic fibrosis stage and inflammatory activity grade. Radiology 258:739-748. https://doi.org/10.1148/radiol.10100853

17. Bakan AA, Inci $E$, Bakan S et al (2012) Utility of diffusion-weighted imaging in the evaluation of liver fibrosis. Eur Radiol 22:682-687. https://doi.org/10. 1007/s00330-011-2295-z

18. Bonekamp S, Torbenson MS, Kamel IR (2011) Diffusion-weighted magnetic resonance imaging for the staging of liver fibrosis. J Clin Gastroenterol 45: 885-892. https://doi.org/10.1097/MCG.0b013e318223bd2c

19. Lewin M, Poujol-Robert A, Boëlle PY et al (2007) Diffusion-weighted magnetic resonance imaging for the assessment of fibrosis in chronic hepatitis C. Hepatology 46:658-665. https://doi.org/10.1002/hep.21747

20. Shayesteh M, Shayesteh A, Motamedfar A et al (2018) The clinical value of the apparent diffusion coefficient of liver magnetic resonance images in patients with liver fibrosis compared to healthy subjects. J Fam Med Prim Care 7:1501. https://doi.org/10.4103/jfmpc.jfmpc_299_18

21. Do RKG, Chandanara H, Felker E et al (2010) Diagnosis of liver fibrosis and cirrhosis with diffusion-weighted imaging: Value of normalized apparent diffusion coefficient using the spleen as reference organ. Am J Roentgenol 195:671-676. https://doi.org/10.2214/AJR.09.3448

22. Ghany MG, Strader DB, Thomas DL et al (2009) Diagnosis, management, and treatment of hepatitis C: an update. Hepatology 49:1335-1374. https:// doi.org/10.1002/hep.22759

23. Puoti C, Guarisco R, Bellis L et al (2009) Diagnosis, management, and treatment of hepatitis C. Hepatology 50:322

24. Verloh N, Utpatel K, Haimerl M et al (2018) DWI - histology: a possible means of determining degree of liver fibrosis? Oncotarget 9:20112-20118. https://doi.org/10.18632/oncotarget.24981

25. Swets JA (1988) Measuring the accuracy of diagnostic systems. Sci Sci 240: 1285-1293. https://doi.org/10.1126/science.3287615

26. Shin MK, Song JS, Hwang SB et al (2019) Liver fibrosis assessment with diffusion-weighted imaging: value of liver apparent diffusion coefficient normalization using the spleen as a reference organ. Diagnostics:9. https:// doi.org/10.3390/diagnostics9030107

\section{Publisher's Note}

Springer Nature remains neutral with regard to jurisdictional claims in published maps and institutional affiliations.

\section{Submit your manuscript to a SpringerOpen ${ }^{\circ}$ journal and benefit from:}

- Convenient online submission

- Rigorous peer review

- Open access: articles freely available online

High visibility within the field

- Retaining the copyright to your article

Submit your next manuscript at $>$ springeropen.com 